\title{
Smart Table Tennis Racket Using a Rubber Mounted Ultrathin Piezoelectric Sensor Array
}

\author{
Takahiro Yamashita* and Takeshi Kobayashi \\ Sensing System Research Center, National Institute of Advanced Industrial Science and Technology, \\ AIST Tsukuba-East, Namiki 1-2-1, Tsukuba, Ibaraki, 305-8564, Japan
}

(Received May 31, 2020; accepted February 10, 2021)

Keywords: piezoelectric, smart sports, strain sensor, table tennis racket, ultrathin

We have newly developed a smart table tennis racket containing a $5 \times 5$ array of ultrathin piezoelectric sensors between the wood paddle and rubber. Since this racket has highly sensitive sensors mounted in the array, not only ball impact localization but also stroke type can be analyzed in detail. This contribution is a pioneering work to realize a MEMS-based sensor system for table tennis rackets, which has the potential to be used in training exercises and games to assist table tennis players during matches and to support athletes in training.

\section{Introduction}

Smart sports equipment enables athletes and amateur sports participants to monitor, track, analyze, and improve their athletic performance and fitness. Smart sports products range from gym machines to running shoes, the important points being that the products can collect data continuously and the player can use them without discomfort. In recent years, various sports rackets with embedded sensors have appeared. Most of them, however, analyze the hit points of a ball from the vibration of the embedded sensors in the handle or at the outer edge of the racket. ${ }^{(1)}$

We previously used ultrathin silicon chips in various applications. ${ }^{(2-5)}$ In this study, we develop a smart table tennis racket that directly measures the strain distribution of the racket when a ball is hit by mounting a flexible printed circuit (FPC) with an ultrathin lead zirconate titanate (PZT)/Si strain sensor array between the wood paddle and rubber of the racket (Fig. 1).

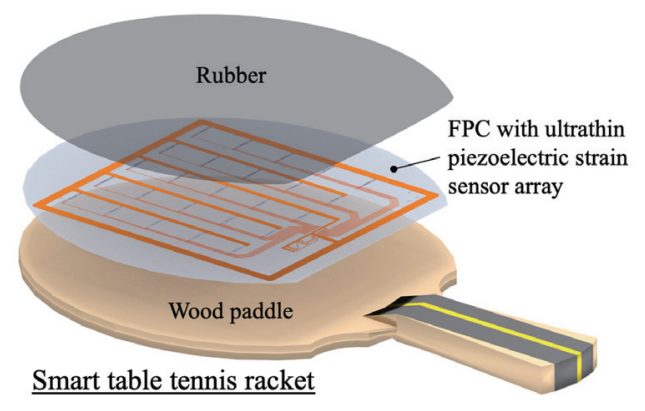

Fig. 1. (Color online) Concept of smart table tennis racket.

*Corresponding author: e-mail: takahiro-yamashita@aist.go.jp https://doi.org/10.18494/SAM.2021.2954 


\section{Fabrication of Smart Racket}

\subsection{FPC with piezoelectric sensor array}

Figure 2 schematically illustrates the fabrication process for the FPC with a strain sensor array. PZT thin films were deposited onto silicon-on-insulator (SOI) wafers and fabricated into ultrathin PZT $(1.9 \mu \mathrm{m}) / \mathrm{Si}(3 \mu \mathrm{m})$ structures by a standard piezoelectric MEMS microfabrication process. ${ }^{(6,7)}$ To transfer printable structures onto an FPC substrate, we fabricated a slightly (six-point) supported ultrathin piezoelectric strain sensor on the handle layer of a 4-inch SOI wafer. First, we prepared a $1.9-\mu \mathrm{m}$-thick PZT layer sandwiched between 100-nm-thick upper and lower Pt/Ti electrodes by sol-gel and sputtering deposition processes on an SOI wafer with a $3-\mu \mathrm{m}$-thick device layer. The total thickness of the piezoelectric structure was approximately $5.1 \mu \mathrm{m}$. Note that the ferroelectric polymer poly(vinylidene difluoride) (PVDF) has been used on flexible devices in many cases because of its high flexibility. ${ }^{(8-10)}$ However, we used PZT because it is a proven ferroelectric material for microfabrication and the MEMS process. ${ }^{(11,12)}$ Then, we patterned and etched the device layer with the piezoelectric structure slightly supported on the handle layer by reactive ion etching of the device, box, and handle layers. By adopting such a slightly supported state, it is possible to separate the sensor from the handle layer and attach it to the flexible substrate using a vacuum collet. Anisotropic etching has been reported as a method of manufacturing an ultrathin sensor structure suitable for a similar transfer printing process, ${ }^{(13)}$ but our approach has the advantage of offering a higher degree of freedom in sensor structure fabrication.

Then the PZT/Si strain sensors were released from the wafer by using a vacuum collet mounted on a chip mounter with an automatic alignment system. The vacuum collet's adsorption power consists of vacuum suction by a vacuum pump. After that, the PZT/Si strain sensors were transfer-printed onto screen-printed 20- $\mu \mathrm{m}$-thick UV-curable resin (Kyoritsu Chemical WORLD ROCK 3970) as an adhesive material on an FPC made of $25-\mu \mathrm{m}$-thick polyimide (PI) as the base film and $\mathrm{Cu}$ as the circuit. After UV curing, the sensor electrodes and $\mathrm{Cu}$ wiring on the PI substrate were connected by conductive paste printing. UV was used to irradiate the UV-curable resin underneath the sensors by the substrate's back side. In the printing process, we performed fine pattern formation by screen printing using silver paste with a high viscosity of approximately $300 \mathrm{~Pa} \cdot \mathrm{s}$ (TOYOCHEM RA FS 089) and ultrahard "nondistortion" stainless-

steel woven wire cloth (Asada Mesh HS-D 650/14) capable of high-viscosity paste printing. ${ }^{(14,15)}$ Note that an insulating paste was printed to prevent short circuits between the upper and lower $\mathrm{Pt} / \mathrm{Ti}$ electrodes before silver paste printing. We can easily integrate such materials onto an FPC by using the technique we developed. Moreover, not only strain sensors but also ultrathin amplifier, MCU, and RF-IC chips can be integrated to realize a fully integrated 2D dynamic strain-sensing sheet. Figure 3 shows the developed $5 \times 5$ (20 mm pitch) ultrathin piezoelectric strain sensor array integrated on a PI FPC. The FPC with the sensor array can be easily installed between the wood paddle and the rubber because its total thickness is less than $100 \mu \mathrm{m}$. 
$\underline{\text { Top view }}$

\section{$\underline{\text { Cross-section view }}$}

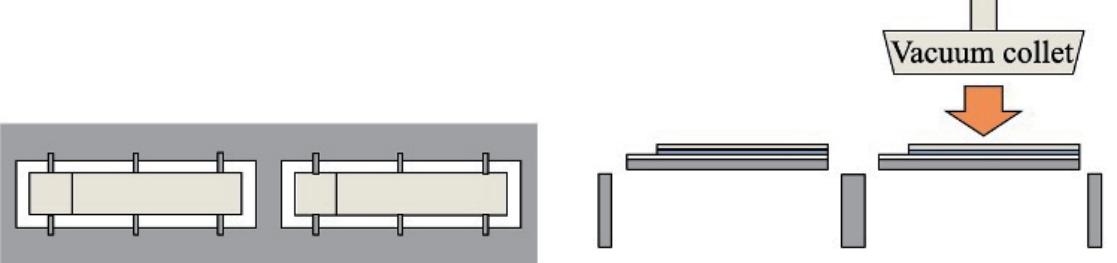

(a) Attach to vacuum collet

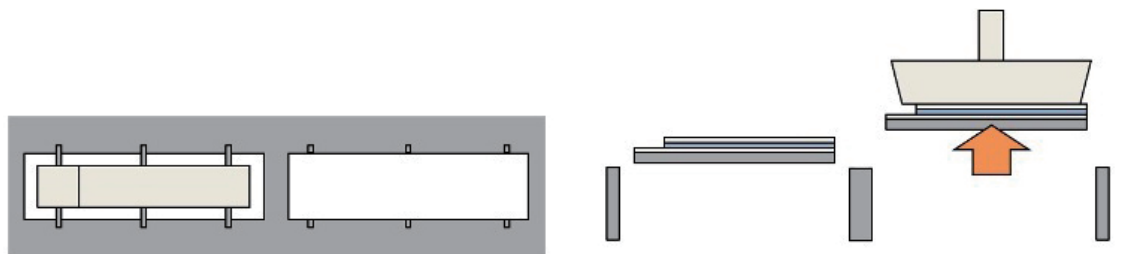

(b) Separate from SOI wafer

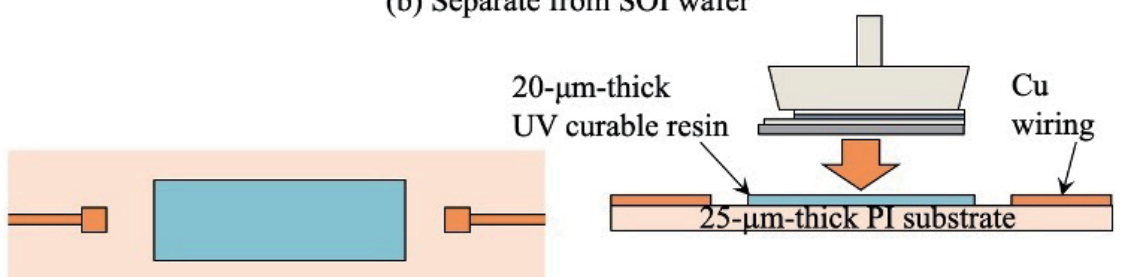

(c) Transfer to flexible substrate

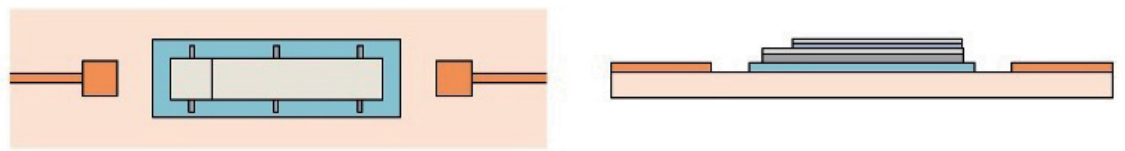

(d) Attach to UV curable resin

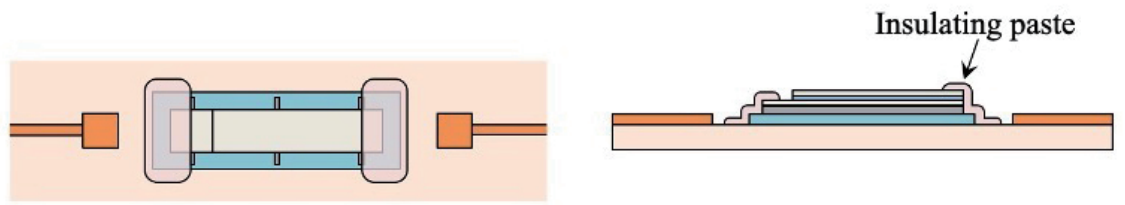

(e) Insulating paste printing
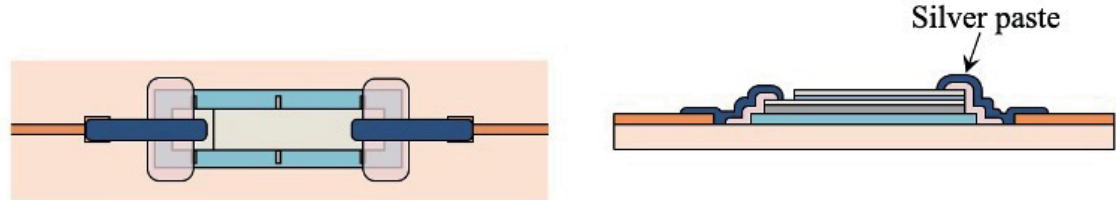

(f) Conductive paste printing

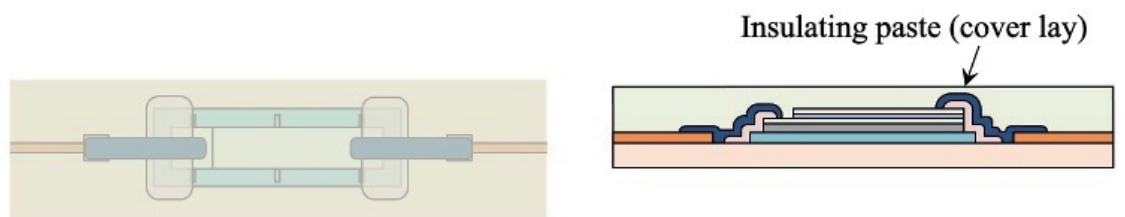

(g) Protective layer printing

Fig. 2. (Color online) Fabrication process for FPC with ultrathin piezoelectric sensor array. 


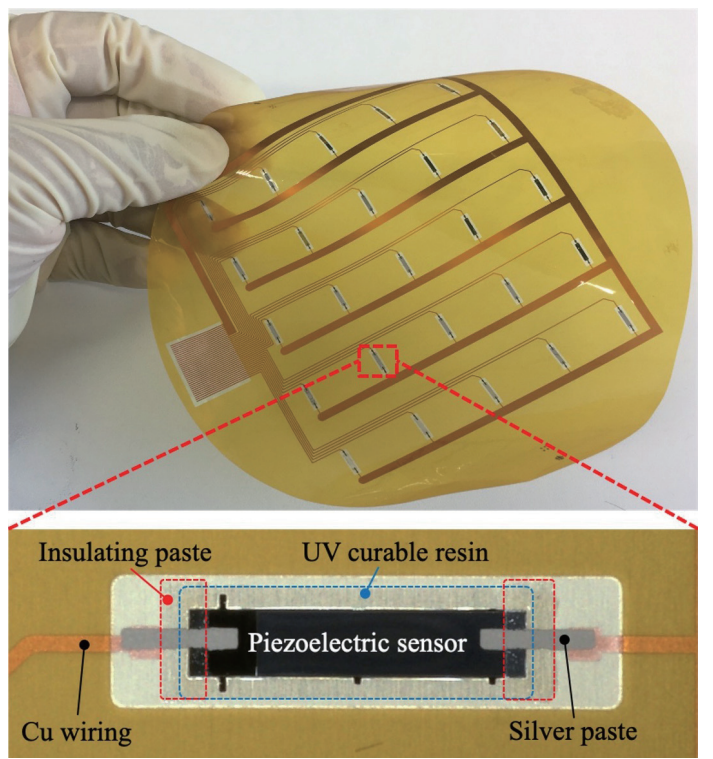

Fig. 3. (Color online) Developed FPC with ultrathin piezoelectric sensor array.

\subsection{Wireless module}

To connect the developed FPC with the sensor array and a wireless module for data transmission, a flexible flat cable (FFC) was thermocompression-bonded to the end of the $\mathrm{Cu}$ wiring of the FPC. The wireless module consists of amplifiers for 25 piezoelectric sensors, a Bluetooth IC, a microcontroller, and a Li-Po battery. Figure 4 shows the rubber of a table tennis racket with a sensor array FPC attached to which a wireless module is connected. In this prototype, the transmitter and Li-Po battery were attached to the outside of the racket, but the size of the transmitter was $48 \times 33 \times 20 \mathrm{~mm}^{3}$, including the plastic case. The size of the grip of the commercial racket used in the experiment was $80 \times 33 \times 24 \mathrm{~mm}^{3}$. Therefore, in terms of volume, the wireless transmitter and battery would fit inside the grip.

\section{Experimental Results and Discussion}

\subsection{Quantitative evaluation of single sensors}

To quantitatively evaluate the performance of single sensors mounted between the paddle and the rubber of the table tennis racket, a table tennis ball was mounted on a load tester (FTN1-13A, Aikoh Engineering Co., Ltd.), as shown in Fig. 5, and repeatedly pressed against the center of the racket with a load of $3 \mathrm{~N}$ at various speeds. The data obtained by the sensors were processed at a sampling frequency of $100 \mathrm{~Hz}$. Figure 6 shows the load applied to the ball and the output voltage generated by the sensor at the center of the sensor array (orange line) and a sensor located next to it (green line) as a function of time. It can be seen that the output voltage varies with the contact speed at the same load. Comparing the two sensor outputs, we can see that the 


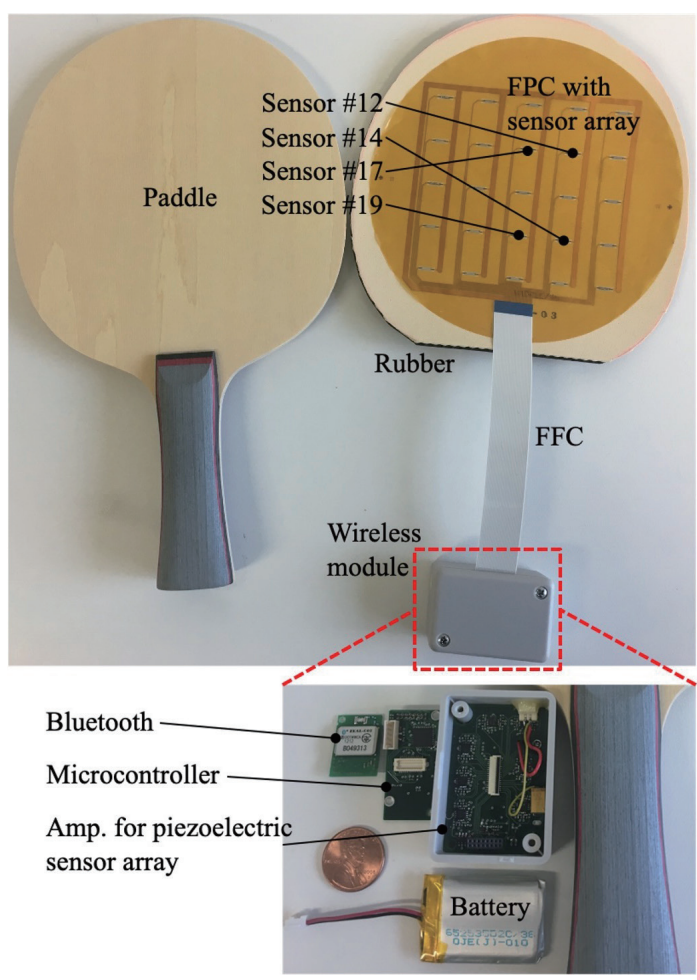

Fig. 4. (Color online) Assembly of smart table tennis racket.
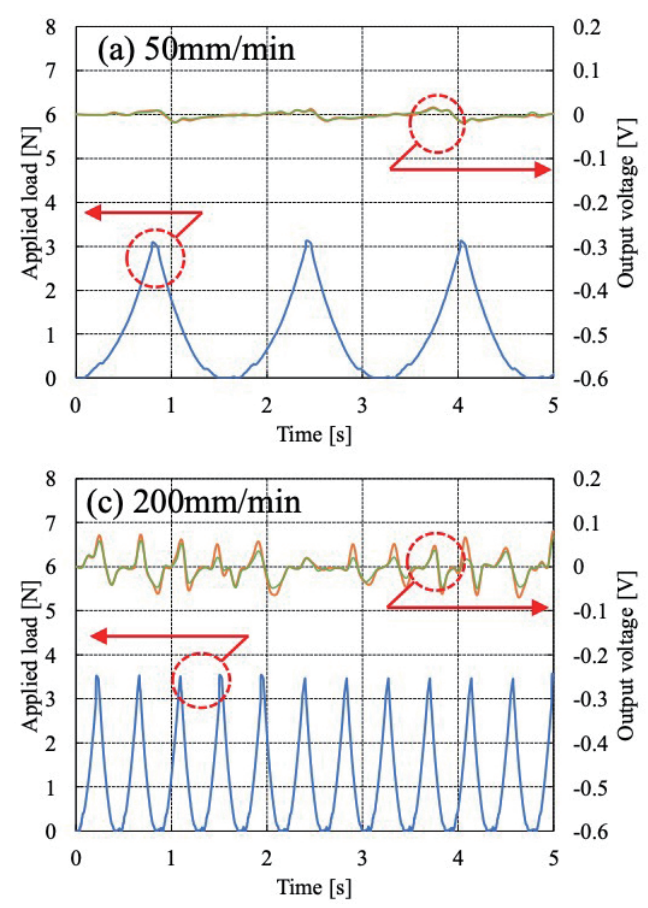

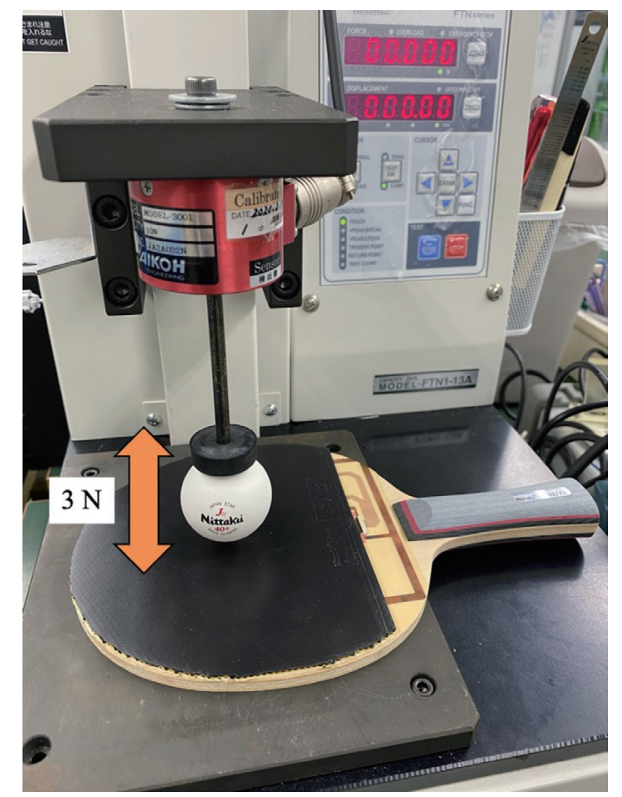

Fig. 5. (Color online) Experimental setup of a ball repeatedly pressed against the center of the racket with a load of $3 \mathrm{~N}$ at various speeds.
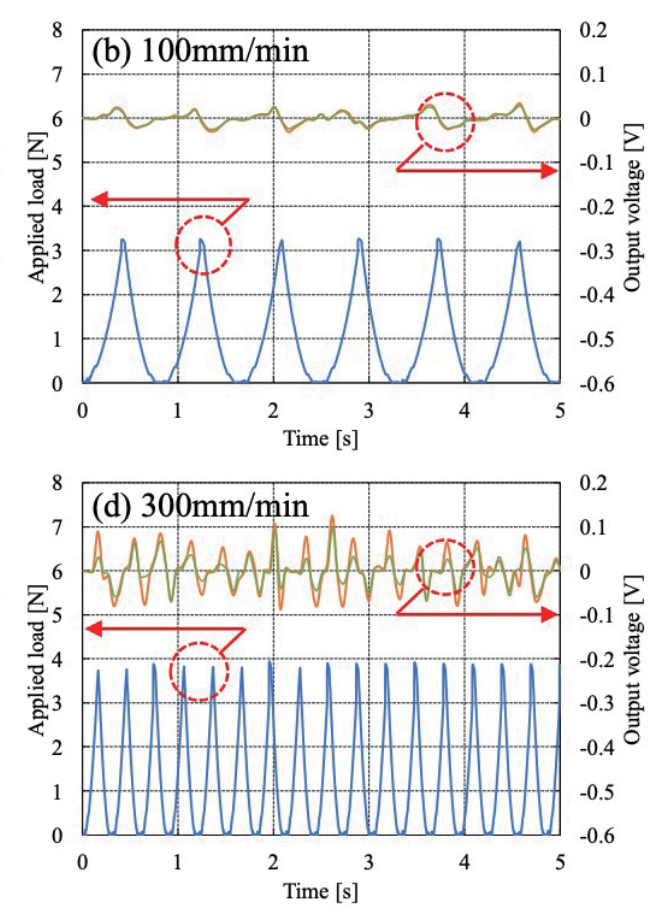

Fig. 6. (Color online) Output voltage generated by the sensor at the center of the sensor array (orange line) and the sensor located next to it (green line) as a function of time when the ball was repeatedly pressed against the center of the racket with a load of $3 \mathrm{~N}$ at various speeds. 
overall behavior is the same, but the magnitude of the output is slightly smaller for the sensor further away from where the ball made contact. Therefore, the impact position can be estimated by evaluating the output distribution of all sensors mounted on the sensor sheet, not just a single sensor output.

Next, the behavior of the sensor was evaluated when the racket was fixed in an upright position, as shown in Fig. 7, and the racket was rubbed with the ball in contact with the surface of the racket while repeatedly moving the ball up and down for $5 \mathrm{~mm}$. Figure 8 shows the applied load on the ball and the output voltage generated at the sensor in the center of the sensor sheet as a function of time, as in Fig. 6. In this experiment, small and noisy output voltages were generated at all speeds, and as the speed of the ball increased, the noisy output voltage also increased, with a large output voltage momentarily generated when the vertical motion was switched. Therefore, evaluating this output voltage may reveal the spin status of the ball.

On the basis of the above results, it was found that the behavior of the output voltage generated by the sensor differed greatly depending on the ball's velocity and the angle at which the ball came in contact with the racket surface. This suggests the prospect of estimating the relationship between the ball impact position and stroke type by evaluating the magnitude of the sensor output and its distribution.

\subsection{Evaluation in game of table tennis}

A racket with a built-in sensor was used in a game of table tennis and the data obtained by the sensor were collected. Figure 9 shows the output voltage for different types of shots and $5 \times 5$ color mappings of the strain distribution obtained from the sensor array. In the case of

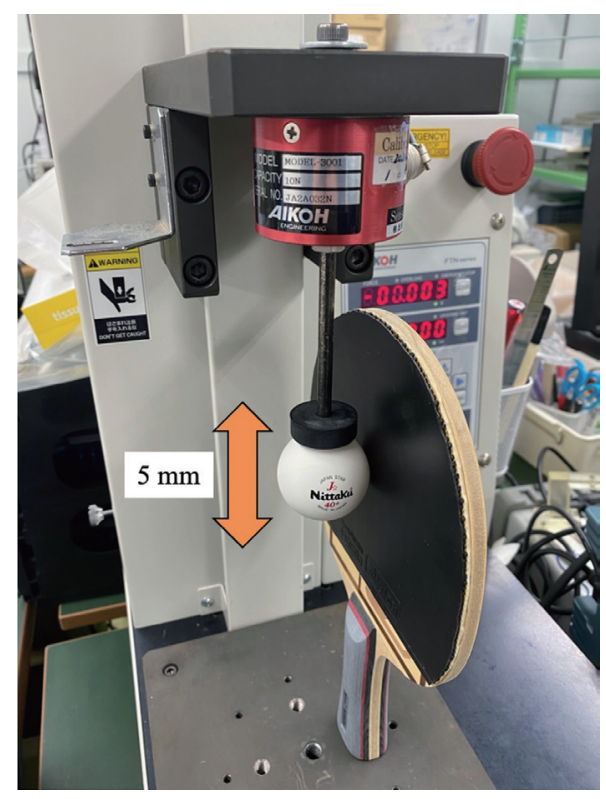

Fig. 7. (Color online) Experimental setup of a ball repeatedly rubbed against the center of the racket with a displacement of $5 \mathrm{~mm}$ at various speeds. 

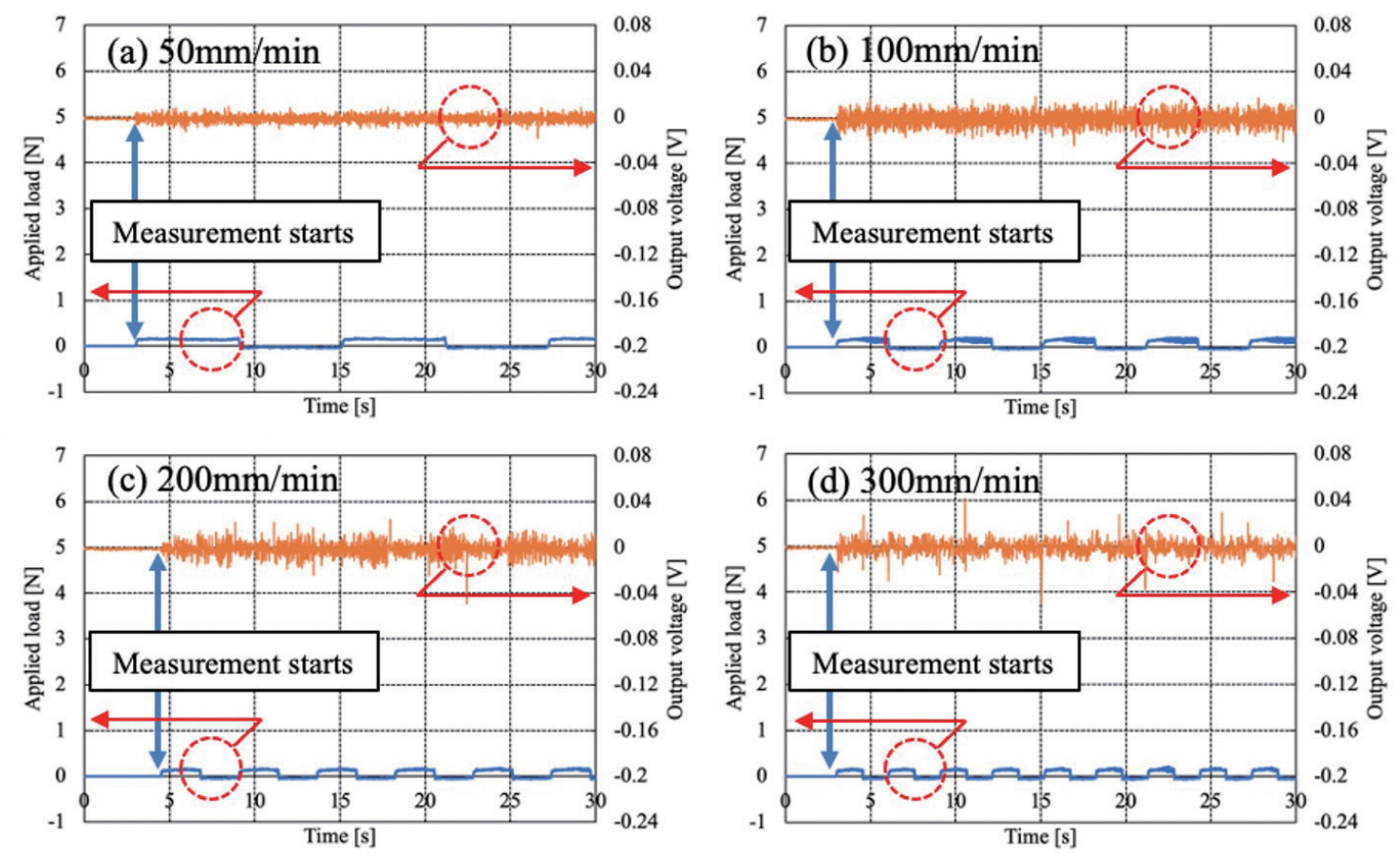

Fig. 8. (Color online) Output voltage generated by the sensor at the center of the sensor array as a function of time when the ball was repeatedly rubbed against the center of the racket with a displacement of $5 \mathrm{~mm}$ at various speeds.
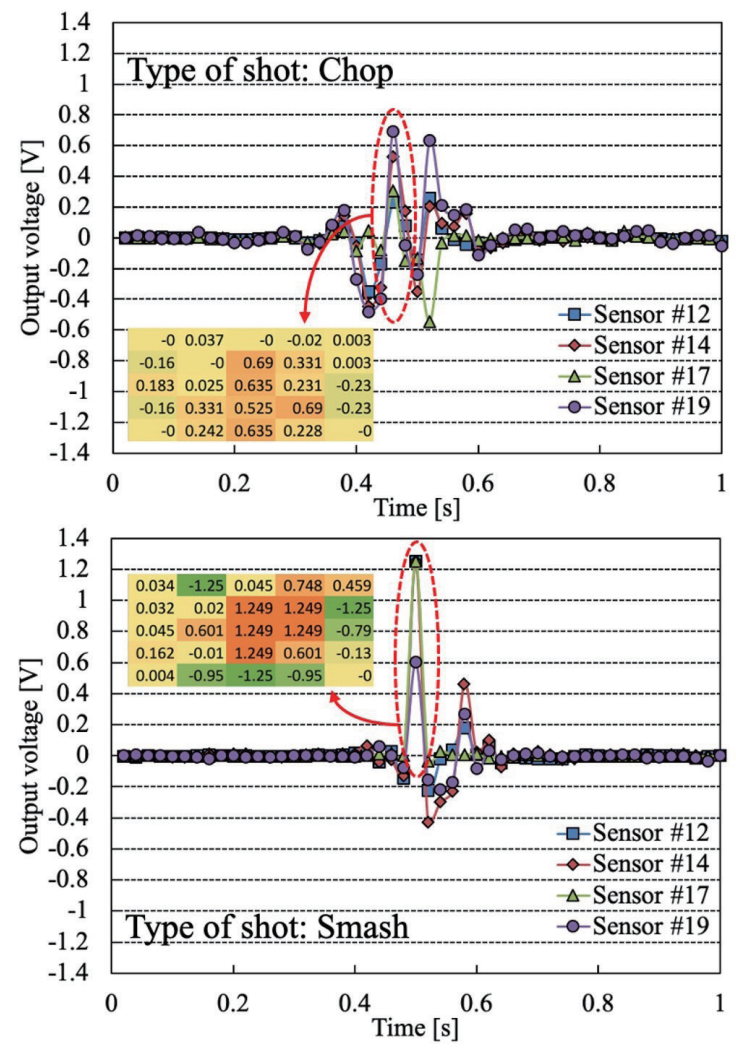

Fig. 9. (Color online) Output voltage for different shots and $5 \times 5$ color mapping of the strain distribution. 
chopping, a relatively loose output voltage was generated over a wide area. On the other hand, when smashing, a sharp peak locally occurred for a short time. From these measurement results, it is confirmed that not only ball impact localization but also the type of shot can be recognized in detail by using this system. Therefore, the trend of ball impact points and shot types under various circumstances of the player can be obtained by analyzing this data.

In the future, we will use a high-speed camera to identify the areas where the ball is hitting the racket and check for consistency with the sensor data. We would also like to install a commercial foil strain gauge in an array on the back of the racket and compare the data obtained with our sensor data.

\section{Conclusions}

We developed a smart table tennis racket containing a $5 \times 5$ array of ultrathin piezoelectric sensors integrated on a PI FPC between the wood paddle and rubber. The developed device has a sensor sheet attached to the rubber of the racket, which makes it easy to manufacture and easy to replace when it breaks. While existing smart sports devices attach vibration sensors to the grip and analyze the vibration results to calculate swing speed and ball speed, this device can directly measure the hitting point and the way the ball is struck. Since this racket has highly sensitive sensors mounted in an array, not only ball impact localization but also stroke type such as a smash or chop can be analyzed in detail. Such rackets with a MEMS-based sensor array system have the potential to be used in training exercises and games to assist table tennis players during matches and to support athlete training.

\section{References}

1 P. Blank, T. Kautz, and B. M. Eskofier: Proc. 2016 ACM Int. Symp. Wearable Computers (ISWC, Heidelberg, 2016) 72-79. https://doi.org/10.1145/2971763.2971778

2 T. Yamashita, S. Takamatsu, H. Okada, T. Itoh, and T. Kobayashi: IEEE Sens. J. 16 (2016) 8840. https://doi. org/10.1109/JSEN.2016.2578936

3 T. Takeshita, T. Yamashita, N. Makimoto, and T. Kobayashi: Jpn. J. Appl. Phys. 56 (2017) 10PF11. https://doi. org/10.7567/JJAP.56.10PF11

4 T. Kobayashi, T. Yamashita, K. Togashi, R. Oohigashi, H. Okada, T. Takeshita, T. Takamatsu, and T. Itoh: Proc. 19th Int. Conf. Solid-State Sensors, Actuators and Microsystems (Transducers 2017) (IEEE, Kaohsiung, 2017) 327-330. https://doi.org/10.1109/TRANSDUCERS.2017.7994055

5 S. Takamatsu, S. Goto, M. Yamamoto, T. Yamashita, T. Kobayashi, and T. Itoh: Sci. Rep. 9 (2019) 1983. https:// doi.org/10.1038/s41598-019-39364-2

6 T. Kobayashi, Y. Suzuki, N. Makimoto, H. Funakubo, and R. Maeda: Proc. IEEE MEMS 2015 (IEEE, Estoril, 2015) 1098-1101. https://doi.org/10.1109/MEMSYS.2015.7051155

7 T. Yamashita, H. Okada, T. Itoh, and T. Kobayashi: Jpn. J. Appl. Phys. 54 (2015) 10ND08. https://doi. org/10.7567/JJAP.54.10ND08

8 S. Takamatsu, T. Kobayashi, T. Imai, T. Yamashita, and T. Itoh: Procedia Eng. 25 (2011) 203. https://doi. org/10.1016/j.proeng.2011.12.050

9 T. Yamashita, S> Takamatsu, T. Kobayashi, and T. Itoh: Proc. 17th Int. Conf. Solid-State Sensors, Actuators and Microsystems (Transducers 2013) (IEEE, Barcelona, 2013) 1561-1564. https://doi.org/10.1109/ TRANSDUCERS.2013.6627080

10 H. Nogami, H. Okada, S. Takamatsu, T. Kobayashi, R. Maeda, and T. Itoh: Jpn. J. Appl. Phys. 52 (2013) 09KD15. https://doi.org/10.7567/JJAP.52.09KD15

11 L. P. Wang, R. Wolf, Q. Zhou, S. Trolier-McKinstry, and R. J. Davis: MRS Proc. 657 (2000) EE5.39. https:// doi.org/10.1557/PROC-657-EE5.39 
12 X. Y. Wang, C. Y. Lee, C. J. Peng, P. Y. Chen, and P. Z. Chang: Sens. Actuators, A 143 (2008) 469. https://doi. org/10.1016/j.sna.2007.11.027

13 C. Dagdeviren, Y. Su, P. Joe, R. Yona, Y. Liu, Y. S. Kim, Y. Huang, A. R. Damadoran, J. Xia, L. W. Martin, Y. Huang, and J. A. Rogers: Nat. Commun. 5 (2014) 4496. https://doi.org/10.1038/ncomms5496

14 T. Yamashita, Y. Zhang, T. Itoh, and R. Maeda: Proc. 16th Test, Integration and Packaging of MEMS/MOEMS (DTIP 2014) (IEEE, Cannes, 2014) 54-57. https://doi.org/10.1109/DTIP.2014.7056662

15 T. Yamashita, Y. Zhang, H. Okada, T. Itoh, and R. Maeda: Proc. IEEE Sensors 2014 (IEEE, Valencia, 2014) 742-745. https://doi.org/10.1109/ICSENS.2014.6985106

\section{About the Authors}

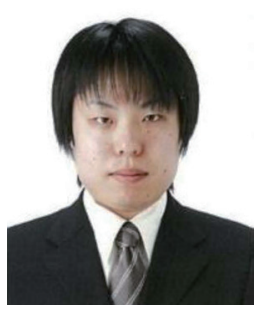

Takahiro Yamashita received his M.E. and Ph.D. degrees in precision engineering from the University of Tokyo, Japan, in 2007 and 2012, respectively. After working as a researcher with the BEANS Laboratory, he became a research scientist with the National Institute of Advanced Industrial Science and Technology (AIST) in 2013. He is currently a senior researcher with the Sensing System Research Center, AIST. His research interests are flexible microsystems involving printed electronics. (takahiro-yamashita@aist.go.jp)

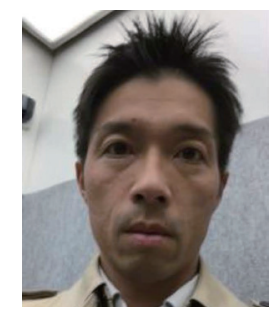

Takeshi Kobayashi received his B.S. and M.S. degrees in materials science from the University of Tokyo. He also received his Ph.D. degree in materials science from the University of Tokyo in 2002. He worked as a researcher in AIST. He is currently the leader of one of the research teams at the Sensing System Research Center, AIST. His research interests include piezoelectric MEMS devices and their application to wireless sensor networks. (takeshi-kobayashi@aist.go.jp) 